\title{
A versatile data logging program in BASIC for the Laptop computer
}

\author{
PAUL S. GRAFF AND BILLY W. HIPP
}

\section{Abstract}

Recording and management of field or laboratory data can be aided through use of a data logging computer. Data are electronically transferred from the data logger to a larger computer for statistical analysis, eliminating re-entry from field sheets and reducing the potential for error. Laptop computers offer advantages over specialized data loggers, but require a software program to function efiiciently as a data logger. Range research uses might include recording plant sample weights, recording livestock weights and condition scores, plant growth measurements, and frequency counts. A flexible data entry program accommodating several experimental designs was written in BASIC for creating analysisready data files, using either keyboard or RS-232 data input.

Key Words: data management, electronic data collection, sampling methods, BASIC computer program

Data collection and data management can be the most arduous aspect of any research study, particularly when projects involve hundreds of experimental units, measured on several occasions. Manual data entry from field sheets is labor intensive, requires error checking, and can slow or stifle ongoing research. Most researchers are aware of the benefits of using specialized data loggers or "electronic notebooks" which are, more or less, hybrids between a hand-held calculator and a personal computer. Use of a data logger allows data to be entered once, at the point of original observation, and subsequently transferred to a personal or mainframe computer for analysis. Data transfer is accomplished using a direct cable connection, or remotely, using a telephone modem. Major disadvantages of many data loggers include cost $(\$ 1,000-4,000)$, cryptic programming languages and operating systems commands, small keyboards with complex multi-function keys, and small video display size.

Authors are research associate and professor, Texas Agr. Exp. Sta., 17360 Coit Road, Dallas 75252-6599.

Report is published with approval of the Director, Texas Agr. Exp. Sta. as TA23225.

Manuscript accepted 4 October 1988.
A battery-operated, portable laptop computer, such as the TRS80 (Tandy Corp. ${ }^{1}$ ), Model 102, can be programmed to perform as a data logger and offers advantages of a typewriter-style keyboard, a $40 \times 8$ character display, programming in the BASIC language, a built-in telephone modem for remote data transfer, and is less expensive (\$499) than many specialized data loggers. Drawbacks include reduced portability relative to smaller data loggers, lack of weather-proofing, extreme memory limitation, and "blacking out" of LCD (liquid crystal diode) displays caused by the heat of direct sunlight (Demment and Greenwood 1987). A major reason the laptop computer has failed to gain wide usage as a data logger is the lack or scarcity of software designed specifically for this purpose (Cooney 1985). Several noncommercial data logging programs have been recently introduced with the objective of meeting the agricultural researcher's needs (Gilreath 1985, Kidger and McNicol 1986, Jackson and Stone 1987). Range research uses might include site inventory, recording "hits" on various plant species, recording plant growth measurements, weighing dried plant samples, and recording livestock weights or behavioral data. Demment and Greenwood (1987) used a portable computer programmed in BASIC, for field recording of livestock feeding behavior in realtime, citing the advantages of the computer over field sheet recording.

The purpose of our work was to provide a highly flexible, menu-driven data logging program for the range or agricultural researcher, easily adaptable to run under variations of Microsoft BASIC, which includes important features either not reported, or not availabile in existing programs. These features include (1) the ability to input data both from the keyboard or from devices (e.g., electronic balances) connected to the computer's RS-232 (serial) port; (2) permanent storage of an experiment's set-up information (i.e., study title, treatment labels, number of reps, number of parameters to measure, and parameter labels) to expedite data

\footnotetext{
'Mention of a trademark or a proprietary product does not constitute a guarantee or warranty of the product by the Texas Agricultural Experiment Station and does not imply its approval to the exclusion of other products that may be suitable.
} 


\begin{tabular}{|c|c|c|c|c|c|c|c|}
\hline $\begin{array}{l}\text { PAN } \\
\text { PAN } \\
\text { PAN } \\
\text { PAN } \\
\text { PAN } \\
\text { PAN } \\
\text { PAN } \\
\text { PAN } \\
\text { PAN } \\
\text { PAN } \\
\text { PAN } \\
\text { PAN } \\
\text { PAN } \\
\text { PAN } \\
\text { PAN } \\
\text { PAN } \\
\text { PAN } \\
\text { PAN } \\
\text { PAN } \\
\text { PAN } \\
\text { PAN }\end{array}$ & $\begin{array}{ll}0 & \mathrm{~N} \\
0 & \mathrm{~N} \\
0 & \mathrm{~N} \\
1 & \mathrm{~N} \\
1 & \mathrm{~N} \\
1 & \mathrm{~N} \\
2 & \mathrm{~N} \\
2 & \mathrm{~N} \\
2 & \mathrm{~N} \\
0 & \mathrm{~N} \\
0 & \mathrm{~N} \\
0 & \mathrm{~N} \\
1 & \mathrm{~N} \\
1 & \mathrm{~N} \\
1 & \mathrm{~N} \\
2 & \mathrm{~N} \\
2 & \mathrm{~N} \\
2 & \mathrm{~N} \\
0 & \mathrm{~N} \\
0 & \mathrm{~N} \\
0 & \mathrm{~N}\end{array}$ & $\begin{array}{l}2 \\
3 \\
1 \\
2 \\
3 \\
1 \\
2 \\
3 \\
1 \\
2 \\
3 \\
1 \\
2 \\
3 \\
1 \\
2 \\
3 \\
1 \\
2 \\
3\end{array}$ & $\begin{array}{l}\text { COLR } \\
1.0 \\
1.0 \\
1.0 \\
1.0 \\
2.0 \\
2.0 \\
3.0 \\
4.0 \\
2.0 \\
2.0 \\
2.0 \\
1.0 \\
3.0 \\
3.0 \\
4.0 \\
4.0 \\
5.0 \\
5.0 \\
2.0 \\
2.0 \\
2.0\end{array}$ & $\begin{array}{l}23.0 \\
15.0 \\
25.0 \\
25.0 \\
30.0 \\
35.0 \\
40.0\end{array}$ & $\begin{array}{l}\text { SMTH } \\
2.0 \\
1.0 \\
1.0 \\
2.0 \\
2.0 \\
2.0 \\
2.0 \\
2.0 \\
2.0 \\
4.0 \\
4.0 \\
3.0 \\
4.0 \\
4.0 \\
3.0 \\
4.0 \\
4.0 \\
3.0 \\
5.0 \\
5.0 \\
5.0\end{array}$ & $\begin{array}{r}\text { ROLL } \\
4.0 \\
2.0 \\
1.0 \\
3.0 \\
3.0 \\
2.0 \\
2.0 \\
2.0 \\
2.0 \\
3.0 \\
3.0 \\
2.0 \\
3.0 \\
3.0 \\
3.0 \\
4.0 \\
4.0 \\
4.0 \\
4.0 \\
5.0 \\
5.0\end{array}$ & $\begin{array}{l}4.0 \\
4.0 \\
4.0 \\
4.0 \\
5.0 \\
5.0 \\
3.0 \\
3.0 \\
3.0\end{array}$ \\
\hline & & & & & . & . & \\
\hline & & & & & & • & \\
\hline & & & 5. & $\& I$ & 5 & 501 & \\
\hline
\end{tabular}

Fis. 1. Sample dataset produced by the data logging program. Treatment labels were entered in the form " $n$ PAN $n N^{\prime \prime}$, specifying 3 replications and 5 parameters to measure.

entry on subsequent collection dates; (3) menu-selected experimental designs, determining the order of data entry, (4) automatic generation of sequential numbers when needed for plot labels, and (5) sorting of datasets in which treatments are randomized.

\section{Description and Specifications}

Major program functions are selected from 2 menus. The first menu presented the user includes the following options: (1) enter a keyboard dataset, (2) produce a dataset from RS-232 input, (3) enter and store a dataset format, (4) resume an interrupted dataset, (5) sort an existing file, (6) show existing files, and (7) exit to operating system. Selecting options 1,2 , or 3 , causes display of a second menu from which a data entry sequence, pertaining to the experiment's design or field layout, is selected. Secondary menu options are: (1) sequential treatments and reps (sampling order is determined by the order of treatment labels stored in the format file); (2) sequential numbers (user provides the beginning number and total experimental units); (3) randomized complete block (user is prompted for each treatment label because of randomization, and program assumes an entire block will be sampled before proceeding to the next); (4) randomized treatments with sequential reps or samples (user supplies the treatment label because of randomization, but sequential prompting for reps or samples occurs automatically); (5) randomized treatments and reps (user must supply both the treatment identification and replication number as a single, composite entry); and (6) return to the initial menu. Menu use reduces user confusion and initialization time, since basic information and assumptions are programmed into each menu item.

Datasets can be produced using the keyboard or the computer's serial port as input sources. Serial input devices might include digital electronic balances, analytical laboratory equipment, or outdoor environmental monitoring equipment.

The program uses dataset "templates" which are files for storing certain dataset information, such as treatment labels, dataset title, and number of replications pertaining to each experiment. The template is created and stored prior to initial data entry for a given experiment. The template can be recalled for sampling on future dates as well, eliminating the need to retype complex or numerous treatment labels. Once a template is created, actual data recording can proceed much more rapidly than the usual paper and pencil method.

Studies with "treatment" labels consisting of sequential numbers are easily accommodated by the program. After selecting "sequential numbers" from the secondary menu, the user is prompted for the total number of plots to measure and the starting number.

The program produces SAS (Statistical Analysis System)' ${ }^{1}$ or other statistics software-compatible datasets in the format:

\section{TREATMENT REP PARAMETER1 PARAMETER2 PARAMETER 3 etc.}

Figure 1 shows a sample dataset created using the program, which would require only minor editing for computer analysis. There is no real limit on the number of parameters that can be entered for each treatment, as long as the computer's line length limit is not exceeded; however, our program limits the number of parameters to 10 . The program automatically right-justifies treatment labels in the dataset and numeric and alpha-numeric data are also rightjustified in columns according to a user-specified format. A column header is printed at the top of each dataset, identifying column contents, and a dataset identification line, current date, and time are stamped at the end of the dataset. Datasets with treatments randomized can be optionally sorted by the program upon completion.

The program code was written to operate with the Microsoft BASIC interpreter supplied with the TRS-80, models 100 and 102 , but can be easily modified to run under IBM ${ }^{\circ}$ or GW-BASIC ${ }^{\circ}$. The minimum RAM requirement is $16-18 \mathrm{~K}$. The TRS-80 version occupies 12,800 bytes in storage, or 11,800 bytes if "REMark" statements are omitted from the BASIC code. The program requires no special hardware to function, with the exception of a null modem adapter for RS-232 input. A diskette (5.25-inch, 360K, MS-DOS format) containing the TRS-80 (interpreted) and IBM, GW-BASIC (interpreted and compiled) versions of the program, and an instructional file covering installation, operation, and customization hints can be obtained from the authors. ${ }^{2}$

\section{Operation}

The program can be installed using a desktop personal computer's text editor to produce the code, and transferred through the serial (RS-232) adapter to the laptop computer, or can be typed directly into the laptop computer using the laptop's text editor or BASIC interpreter. A typical data entry session for a new experiment would include the following steps: (1) select "Enter a Template" from the primary menu; (2) a data entry sequence is selected from the secondary menu, such as "Sequential Treatments", and the user answers program prompts for study title, number of treatments, replications, parameters to measure, etc., to produce the dataset template file; (3) program returns to the main menu and user selects "Keyboard Dataset" or "RS-232 Dataset"; (4) user is again presented the secondary menu and selects "Sequential Treatments" (the program redundantly displays the secondary menu to allow maximum flexibility in data entry order); (5) the

2Send request to: Paul Graff, Texas Agricultural Experiment Station, 17360 Coit Rd., Dallas, TX 75252-6599. A \$10 (U.S.) check or money order, payable to Texas Agricultural Experiment Station, is requested to cover the cost of the diskette, postage, and packaging. 


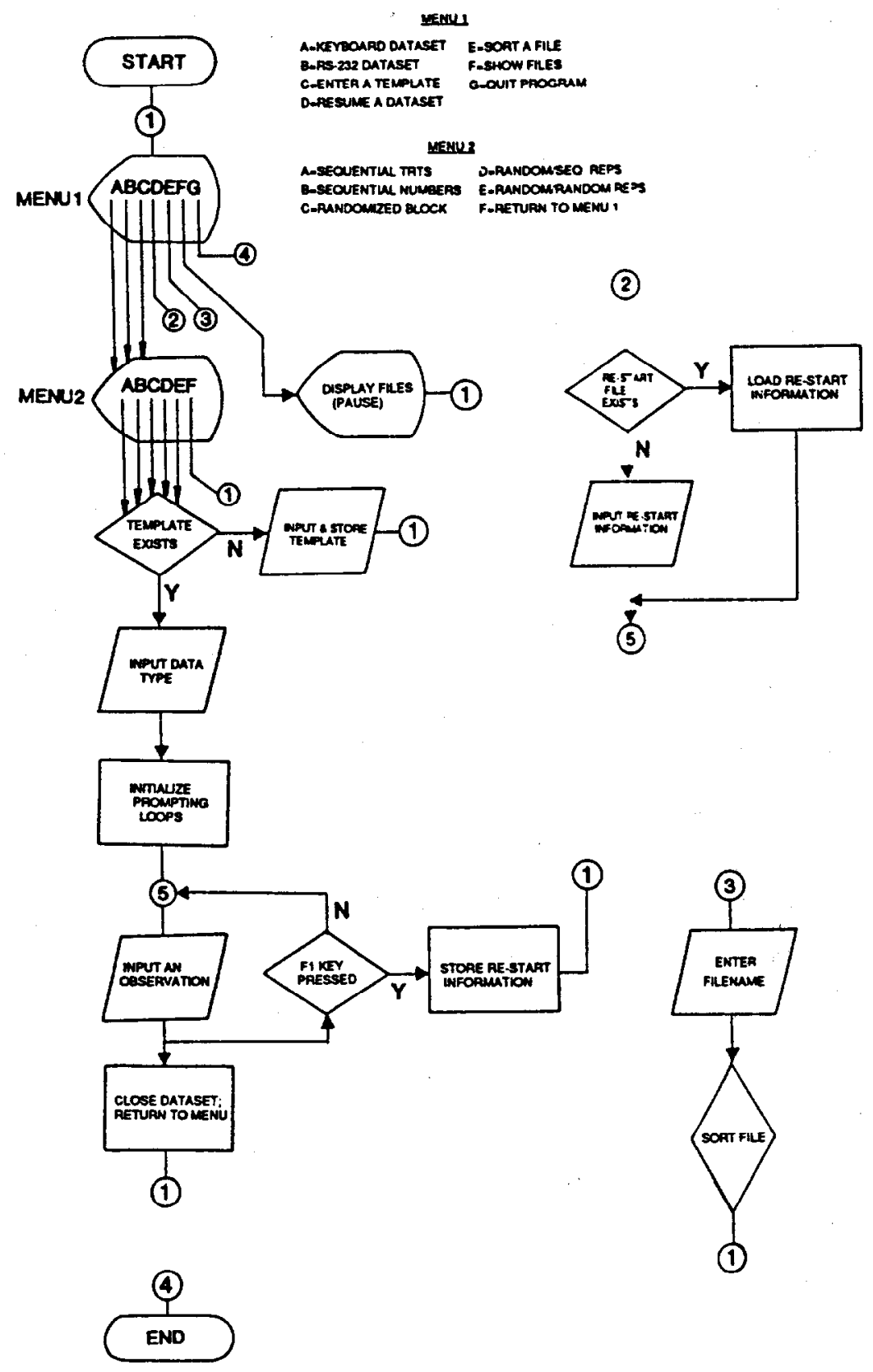

Fis. 2. Abbreviated flowchart of data logging program execution.

program prompts for the type of data (numeric of alpha-numeric), and the expected width of a data column; (6) data entry proceeds can be interrupted with a function key); and (7) program returns to main menu. Subsequent sessions would not require steps 1 and 2 .

Data entry can be interrupted by striking a programmed function key and automatically resumed at a later time, without having to re-enter any setup information. In the event of unplanned, interrupted entry caused by battery failure, a dataset can be completed by specifying the number of completed treatments to skip, and appending to the partially completed dataset. Figure 2 provides an abbreviated flowchart of program execution. A future version of the program will allow use of a bar code reader for scanning treatment names, allow inclusion of a date identifier column in the dataset, and provide menu options for dataset printing or electronic transfer (RS-232).

\section{Literature Cited}

Cooney, T.M. 1985. Portable data collectors, and how they're becoming useful. J. Forest. 83:18-23.

Demment, M.W., and G.B. Greenwood. 1987. The use of a portable computer for the real-time recording of observations of grazing behavior in the field. J. Range Manage. 40:284-285.

Gilreath, J.P. 1985. Description of a basic program for data collection using a portable microcomputer. Hort. Science. 20:301.

Jackson, H.O, and J.A. Stone. 1987. Improved data collection program for a notebook-size computer. Agron. J. 79:1087-1089.

Kidger, R., and J.W. McNicol. 1986. A program for data recording on a portable microcomputer. Computers and Electronics in Agriculture. 1:213-217. 